\title{
El servicio de Medicina complementaria de EsSalud, una alternativa en el sistema de salud peruano
}

\author{
Complementary Medicine Service in EsSalud, an alternative in the Peruvian Health System
}

\section{Sr. Editor:}

Se conoce como Medicina alternativa y complementaria (MAC) al conjunto de prácticas de atención de salud que no están integradas en el sistema sanitario convencional ni son parte de la propia tradición de un país y cuya utilidad ha sido demostrada científicamente (1).

La MAC posee ciertos beneficios frente a la medicina convencional, como son su bajo costo, bajo riesgo (si es utilizada adecuadamente), visión holística, humanitaria e individualizada del paciente, y mejor efecto terapéutico y paliativo en enfermedades crónicas y terminales (1).

El uso de MAC va en aumento alrededor del mundo. Se estima que el uso de la MAC es del $49 \%$ en Francia y $70 \%$ en Canadá (2). En el Perú, un estudio realizado en un hospital de Lima indicó que aproximadamente el $70 \%$ de los pacientes la utilizó alguna vez (2) y otro estudio en hospitales públicos de provincia, estimó que el $40,4 \%$ de los usuarios conocen alguna terapia de MAC y el 33\% la ha utilizado alguna vez (3).

De acuerdo a la estrategia de la Organización Mundial de la Salud (OMS) sobre Medicina tradicional (1), es importante diseñar e implementar políticas que adecúen la oferta de estos servicios a las necesidades de la población. Para ello, se recomienda la incorporación de la MAC a los sistemas de salud nacionales (4).

Esta incorporación ha sido llevada a cabo de manera satisfactoria en varios países, como Francia, Alemania, Estados Unidos, México, Brasil, Colombia, Argentina y Chile $(1,4)$, mientras que en otros aún está en proceso o aún no ha comenzado. Esta demora puede deberse a una escasa aceptación de la MAC por parte de autoridades y profesionales de la salud, el desconocimiento de sus beneficios y problemas de coordinación entre las entidades pertinentes (4).

En Perú, existe un servicio de Medicina complementaria implementado en EsSalud (SMCEsSalud), el cual aún no es muy conocido entre los profesionales de la salud ni entre el público en general, siendo el objetivo de la presente comunicación difundir su importancia, sus actividades y sus beneficios.

El SMC-EsSalud fue creado en 1998 y a la fecha cuenta con 55 locales distribuidos en tres niveles de atención, que ofrecen una gran variedad de terapias (5), como se detalla en la tabla 1.

Anualmente se atienden unos 50000 asegurados en el SMC-EsSalud y desde su creación se han beneficiado más de 500000 pacientes. Entre los logros de este servicio podemos mencionar: un ahorro institucional de más de 25 millones de soles para la institución, tener presencia en las 29 redes asistenciales de EsSalud, una satisfacción del paciente del 92\%, disminución del consumo de medicamentos convencionales en $19 \%$ y $22 \%$ de pacientes en los CAMEC y UMEC respectivamente, reducción del consumo de analgésicos en $80 \%$, disminución del uso de broncodilatadores en $60 \%$, y eliminación del consumo de ansiolíticos y antidepresivos (5).

El SMC-EsSalud aún es poco conocido y a veces visto con recelo por pacientes y médicos, por lo cual es necesario que este servicio se preocupe por difundir sus actividades y beneficios por diversos medios de comunicación. Además puede ofrecer rotaciones clínicas y talleres de capacitación en terapias de MAC para estudiantes y profesionales de la salud.

Finalmente, resulta necesario que otras entidades prestadoras de servicios de salud (como el Ministerio de Salud y el Sistema Metropolitano de la Solidaridad) 
Tabla 1. Distribución de los locales de EsSalud que ofrecen el servicio de Medicina complementaria en Perú.

\begin{tabular}{|c|c|c|c|c|}
\hline $\begin{array}{l}\text { Nivel de } \\
\text { atención }\end{array}$ & Servicios & Terapias que se ofrecen & Distribución & $\begin{array}{l}\text { Total de } \\
\text { locales }\end{array}$ \\
\hline Primer nivel & $\begin{array}{c}\text { Unidades de Medicina } \\
\text { Complementaria (UMEC) }\end{array}$ & $\begin{array}{l}\text { Trofoterapia } \\
\text { Fitoterapia } \\
\text { Auriculoterapia } \\
\text { Pisocinéticas } \\
\text { Meditación }\end{array}$ & $\begin{array}{c}\text { En todos los } \\
\text { departamentos del } \\
\text { Perú }\end{array}$ & 25 \\
\hline Segundo nivel & $\begin{array}{l}\text { Centros de Atención de } \\
\text { Medicina Complementaria } \\
\text { (CAMEC) }\end{array}$ & $\begin{array}{l}\text { Acupuntura } \\
\text { Medicina Natural } \\
\text { Terapias Manuales } \\
\text { Medicina Energética } \\
\text { Medicina Cuerpo-mente }\end{array}$ & $\begin{array}{l}\text { En todos los } \\
\text { departamentos del } \\
\text { Perú }\end{array}$ & 29 \\
\hline Tercer nivel & $\begin{array}{c}\text { Unidad de Cuidados Paliativos } \\
\text { de Medicina Complementaria } \\
\text { (UCPMEC) }\end{array}$ & $\begin{array}{l}\text { Acupuntura } \\
\text { Masoterapia } \\
\text { Terapia floral } \\
\text { Fitoterapia } \\
\text { Actividad psicocinéticas } \\
\text { (Yoga-Tai chi) }\end{array}$ & $\begin{array}{l}\text { Hospital Edgardo } \\
\text { Rebagliati (Lima) }\end{array}$ & 1 \\
\hline
\end{tabular}

incluyan formalmente entre sus servicios a la MAC, beneficiando a los pacientes que no están asegurados por EsSalud.

\section{EImer Luján-Carpio ${ }^{1,2, a}$, Zaida Lizarraga- Castañeda $^{1,2, a}$, Alexander Mayor-Vega ${ }^{1,2, a}$, Karola Medrano-Canchari ${ }^{1, a}$, Henry Medina-Salazar ${ }^{1,2, a}$, Sergio Goicochea-Lugo ${ }^{1,2, a}$}

\section{Correspondencia:}

Sergio André Goicochea Lugo

Correo electrónico:sgoicochealugo@gmail.com

\section{REFERENCIAS BIBLIOGRÁFICAS}

1. Organización Mundial de la Salud. Estrategia de la OMS sobre medicina tradicional 2014-2023. Ginebra: Organización Mundial de la Salud; 2013. Citado en marzo del 2013 Disponible en: http://apps.who.int/ medicinedocs/documents/s21201es/s21201es.pdf

2. Pena A, Paco O. Medicina alternativa: intento de análisis. An Fac Med. 2007; 68 (1): 87-96.
3. Valenzuela F. Oferta y demanda de servicios de salud con terapias alternativas y complementarias en los hospitales del Ministerio de Salud en las ciudades de Arequipa, Lambayeque y Lima - 2006. Lima: Instituto nacional de Salud; 2006 (Citado el 19 de octubre del 2010). Disponible en: http://www. ins.gob.pe/repositorioaps/0/7/jer/censi_ofer_estu/ RESUMEN_OFERTA_DEMANDA_MAC_2006. pdf

4. Nigenda G, Mora-Flores G, Aldama-López S, Orozco-Núñez E. La práctica de la medicina tradicional en América Latina y el Caribe: el dilema entre regulación y tolerancia. Salud Publica Mex. 2001; 43(1): 41-51.

5. EsSalud. En EsSalud, con medicina complementaria se disminuye hasta en $100 \%$ el uso de antidepresivos. Lima: ESSALUD. (Citado 25 de enero de 2014). Disponible en: http://www.essalud.gob.pe/2013/05/06/ en-essalud-con-medicina-complementaria-sedisminuye-hasta-en-100-el-uso-de-antidepresivos/

Recibido: 22/04/2014 